\title{
Use of diagnostic tests and the appropriateness of the treatment decision in patients with suspected urinary tract infection in primary care in Denmark - observational study
}

Gloria Córdoba ${ }^{* *}$, Anne Holm, Tina Møller Sørensen², Volkert Siersma ${ }^{1}$ Håkon Sandholdt ${ }^{1}$, Marjukka Makela ${ }^{3}$, Niels Frimodt-Møller ${ }^{4}$ and Lars Bjerrum ${ }^{1}$

\begin{abstract}
Background: Inappropriate prescription of antibiotics is the leading driver of antimicrobial resistance (AMR). The majority of antibiotics are prescribed in primary care.

Understanding how general practitioners (GPs) use diagnostic tests and the effect on treatment decision under daily practice conditions is important to reduce inappropriate prescription of antibiotics. The aim of the study was to investigate the use of diagnostic tests in primary care patients with suspected urinary tract infection (UTI) and to assess the appropriateness of the treatment decision (TD) under daily practice conditions in Denmark.

Methods: Prospective observational study. Symptomatic adult patients consulting general practice with suspected UTI recruited over 12 months. The diagnostic workup was registered in a standardized form. The appropriateness of the TD was assessed based on the results of a culture performed at a reference microbiological laboratory. TD was considered appropriate if a patient had a positive culture and was prescribed antibiotics or had a negative culture and was not prescribed antibiotics. TD was considered inappropriate if a patient had a negative culture and was prescribed antibiotics (overtreatment) or had a positive culture and was not prescribed antibiotics (undertreatment).

Results: Four hundred and eighty-eight patients were included. Dipstick was used in $98 \%$ of the patients and urine culture was used in $89 \%$ of the patients; 317 had the culture performed in practice and 117 had the culture performed at the hospital. The appropriateness of the final TD was significantly $(p=0.04)$ lower in patients without culture (55\%) than in patients with culture performed in practice (71\%) or at hospital (69\%).

Conclusion: In a context with wide availability of diagnostic tests, GPs use diagnostic tests for the decision-making process in all patients with suspected UTI. Urine culture is used in the majority of the patients and is associated with a higher proportion of appropriate treatment decisions. Performance of urine culture is therefore important in reducing inappropriate antibiotic prescribing in patients with suspected UTI seeking care in general practice in Denmark.
\end{abstract}

Trial registration: ClinicalTrials.gov NCT02249273.

Keywords: Treatment decision, Point-of-care systems, Urinary tract infections, Anti- bacterial agents, Primary care, Diagnostic test

\footnotetext{
* Correspondence: gloriac@sund.ku.dk

${ }^{1}$ The Research Unit for General Practice and Section of General Practice,

Department of Public Health, University of Copenhagen, Øster Farimagsgade

5, 1014 Copenhagen, Denmark

Full list of author information is available at the end of the article
}

(c) The Author(s). 2018 Open Access This article is distributed under the terms of the Creative Commons Attribution 4.0 International License (http://creativecommons.org/licenses/by/4.0/), which permits unrestricted use, distribution, and

reproduction in any medium, provided you give appropriate credit to the original author(s) and the source, provide a link to the Creative Commons license, and indicate if changes were made. The Creative Commons Public Domain Dedication waiver (http://creativecommons.org/publicdomain/zero/1.0/) applies to the data made available in this article, unless otherwise stated. 


\section{Background}

Optimizing the use of antimicrobials and increasing the investment in diagnostic tests are part of the key strategies of the World Health Organization (WHO) for counteracting the spread and development of antimicrobial resistance (AMR) [1].

Denmark has a low prescription rate of antimicrobials at primary health care level in comparison to other European countries [2]. Nonetheless, there is still a high use of antibiotics at primary health care level [3].

In Denmark, suspected Urinary tract infection (UTI) is a frequent cause for antibiotic prescribing [4] and use of point-of-care tests (POCTs) [5]. There are no national guidelines on the diagnostic procedures to be followed in patients with suspected UTI. It is up to the GPs as managers/owners of the practice to decide the type of urine tests that are available in their practice. Furthermore, it is up to the GPs as clinicians to decide whether a diagnostic test should be performed in a patient with suspected UTI.

There is wide availability of diagnostic tests, which can be used in patients with suspected UTI. The diagnostic workup may include 1) dipstick, 2) microscopy, 3) culture performed and interpreted in practice, and/or 4) culture sent to a hospital laboratory. All practices can send urine samples for culture at hospital and most practices have a small laboratory where they can perform simple testing. GPs receive a fee for performing tests in practice, and there is no economic restriction to the number of tests per individual patient.

The use of urine culture implies a two-step treatment decision: first a decision when the patient consults the GP (index consultation), and a second step after the result of the urine culture is available. For cultures performed in practice, the results can be seen 1 day after the index consultation, while it may take up to 3 or 4 days to receive the results from a hospital.

In busy primary care, diagnostic test results may not translate directly to treatment decisions $[6,7]$, as these decisions are affected by several contextual factors [8,9]. Therefore, the impact of diagnostic workup on appropriate treatment should be assessed under daily practice conditions.

The aim of this study was to describe the use of diagnostic tests in patients with suspected UTI and to assess the impact of the diagnostic work-up on the treatment decision under daily practice conditions in Denmark.

\section{Methods}

\section{Design and setting}

The study protocol has been published previously [10]. An observational study of the management of patients with suspected UTI in general practice under daily practice conditions was carried out between December 2014 and December 2015. It was part of the context assessment of a quality improvement program aimed at reducing the inappropriate prescription of antibiotics in patients with suspected UTI.

\section{Study population and data collection}

A random sample of 500 practices from the capital region of Copenhagen was invited by letter to participate; 39 accepted the invitation. Each practice included up to 20 patients fulfilling four criteria: a) > 18 years of age, b) presence of dysuria and/or frequency, c) suspected UTI, d) not currently taking antibiotics. In a standardized form the GPs registered number of days with symptoms, types of symptoms, potential risk factors for UTI, diagnostic workup performed, and treatment decision.

\section{Urine sample - Reference standard}

All patients were requested to provide a mid-stream urine sample (approx. $20 \mathrm{ml}$ ) of which half could be used for diagnostic purposes in practice, and the other half (reference standard) was cultured at a reference laboratory (Statens Serum Institute, SSI). The sample analysed by SSI was preserved in boric acid and sent by certified post on the day of the consultation. Results from the reference standard analysis were not available for the participating GPs.

At SSI, cultures were analyzed by a microbiology technician who had no information about the clinical history of the patient. A positive culture was defined as growth of $\geq 10^{3}$ colony forming units per milliliter $(\mathrm{CFU} / \mathrm{ml})$ for $E$. coli and $S$. saprophyticus, $\geq 10^{4} \mathrm{CFU} / \mathrm{mL}$ for other typical uropathogens and $\geq 10^{5} \mathrm{CFU} / \mathrm{ml}$ for possible uropathogens according to the European Urinalysis Standards [11].

\section{Definition of variables}

As the main focus of analysis was the role of diagnostic tests in the decision-making process, treatment decision was selected as the primary outcome [10]. The appropriateness of the treatment decision was evaluated against the reference standard (i.e. culture at SSI). Treatment decision was considered as appropriate when a patient with a positive reference culture was prescribed antibiotics or a patient with a negative culture received no antibiotics. Treatment decision was considered as inappropriate for patients with a negative culture who received antibiotics (overtreatment) or patients with a positive culture who were not prescribed antibiotics (undertreatment).

UTI was defined as complicated if the patient was a man, a pregnant woman, a woman $\geq 65$ years, had a relevant co-morbidity or was assessed by the nurse or GP to have complicated cystitis or pyelonephritis. An anamnestic score was calculated. It is the sum of positive findings recorded by the GP, in the standardized data collection form, during the anamnestic phase of the consultation (range 1-11). 
The diagnostic pathways were categorized into three groups: a) no urine culture performed, b) urine culture performed in practice, and c) urine culture performed at the hospital.

\section{Statistical analysis}

The statistical analysis was performed in two steps. First, possible determinants for the use of the various diagnostic tests were investigated in logistic regression models. We tested the following predictor variables individually with practices as a random intercept. The predictors were: a) anamnestic information, b) availability of diagnostic tests and c) information from any diagnostic tests previously performed (See Additional file 1: Table S1).

Secondly, we used a permutation test to assess the impact of the diagnostic pathway variable on the treatment decision. The multinomial regression model was adjusted for the predictors that were significant in the first model and the differences in baseline characteristics of the diagnostic pathway groups. All analyses were performed in the $\mathrm{R}$ programming language and environment v3.3.2 "Sincere Pumpkin Patch" using the lme4 and nnet package [12].

\section{Ethical aspects}

The study was approved by the ethical committee of the Capital region (case number: H-4- 2014-097). Informed written consent was obtained from all patients participating in the study.

\section{Results}

\section{Diagnostic workup}

Four hundred and eighty-eight patients were recruited to the study. Dipstick was done in 480 (98\%) patients and microscopy in 160 (32\%) patients. 434 (89\%) patients had a urine culture performed as part of the diagnostic work-up; done in practices in 317 (65\%) and sent to hospital in $117(24 \%)$ patients. The most common combination of diagnostic tests was dipstick and culture done in practice ( $n=240,49 \%$ of the patients) - Table 1 .

Dipstick results $(n=480)$ included reactions for nitrite and leucocytes. In 334 (69\%) patients, the two reactions pointed in different directions: most frequently, the reaction for leucocytes was positive and for nitrite negative. $63(13 \%)$ patients had negative reactions for both nitrite and leucocytes and 91 (18\%) were positive for both.

Microscopy results $(n=160)$ included identification of $\geq 1$ bacteria per High Power Field (HPF) in 106 (66\%) patients; and identification of $\geq 1$ white blood cell per HPF in 70 (43\%) patients. Combination of $\geq 1$ bacteria $+\geq 1$ white blood cell per HPF in 30 (19\%) patients.

The sensitivity of urine culture performed and interpreted in practice was $94 \%$ (95\% CI 89\%; 96\%), specificity 51\% (95\%CI 43\%; 59\%), Positive Predictive Value - PPV
Table 1 Distribution of the use of diagnostic tools and diagnostic pathways

\begin{tabular}{ll}
\hline & Total $(n=488)$ \\
\hline Dipstick & 480 \\
Microscopy & 160 \\
Culture in practice & 317 \\
Culture sent to the hospital & 117 \\
Diagnostic pathways & \\
No urine culture $(n=54)$ & \\
Dipsticks & 14 \\
Microscopy & 3 \\
Dipsticks + microscopy & 37 \\
Urine culture in practice $(n=317)$ & \\
Dipsticks + urine culture in practice & 240 \\
Dipsticks + microscopy + urine culture in practice & 76 \\
Urine culture in practice & 1 \\
Urine culture sent to the hospital $(n=117)$ & \\
Dipsticks + urine culture in hospital & 73 \\
Microscopy + urine culture in hospital & 4 \\
Dipsticks + microscopy + urine culture in hospital & 40 \\
\hline
\end{tabular}

69\% (95\% CI 63\%; 74\%), Negative Predictive Value - NPV 88\% (95\% CI 79\%;93\%) - see Additional file 1: Table S2.

The sensitivity of urine culture performed at hospital was 90\% (95\% CI 80\%; 95\%), specificity 89\% (95\% CI 78\%;94\%), PPV 90\% (95\% CI 80\%;95\%), NPV 89\% (95\% CI 78\%;94\%) - see Additional file 1: Table S3.

Table 2 shows the distribution of baseline characteristics in patients without culture, those where culture was done in practices and patients whose culture was sent to hospital. The proportion of uncomplicated UTIs was higher (70\%) in patients with no culture performed than in patients with practice culture (60\%) or hospital culture (34\%).

Identification of predictors for use of additional diagnostic testing is shown in Additional file 1: Table S1. Neither a suspected complicated UTI nor the results of dipstick or microscopy predicted the incorporation of urine culture within the diagnostic workup. The incorporation of a diagnostic test was predicted by the availability of diagnostic tests. For example, availability of a microscope in practice decreased the propensity of performing a culture in practice (OR $0.04,95 \%$ CI $0.004 ; 0.5$ ) and availability of culture in practice decreased the propensity of performing a culture at hospital (OR 0.01, 95\% CI 0.001; 0.08).

The incorporation of a diagnostic test was predicted as well by anamnestic information. For example, a high anamnestic score decreased the likelihood of performing urine culture in practice OR 0.7 (95\% CI 0.6; 0.9), but increased cultures at hospital OR 1.5 (95\% CI 1.1; 1.9).

Offensive smell increased urine cultures at hospital OR 2.9 (95\% CI 1.2; 7), but not in practice. 
Table 2 Patients' baseline characteristics and diagnostic pathways

\begin{tabular}{|c|c|c|c|}
\hline & No culture $(n=54)$ & $\begin{array}{l}\text { Culture performed } \\
\text { in practice }(n=317)\end{array}$ & $\begin{array}{l}\text { Culture performed at } \\
\text { hospital }(n=117)\end{array}$ \\
\hline Prevalence confirmed UTI & $29(54)$ & 170(46) & $62(52)$ \\
\hline Uncomplicated UTI & $38(70)$ & $191(60)$ & $41(34)$ \\
\hline Women & $51(94)$ & 293(92) & 110(94) \\
\hline Younger patients ( $<=64$ years) & $41(76)$ & 218(69) & $88(75)$ \\
\hline Number of days with symptoms (Median-IQR) & $2(1 ; 4)$ & $2(1 ; 5)$ & $2(1 ; 4)$ \\
\hline \multicolumn{4}{|l|}{ Signs \& Symptoms } \\
\hline Dysuria & $38(70)$ & $214(67)$ & $88(75)$ \\
\hline Frequency & $38(70)$ & $211(66)$ & $84(72)$ \\
\hline Urgency & $24(44)$ & $152(48)$ & $73(62)$ \\
\hline Suprapubic pain & $17(31)$ & $85(27)$ & $45(38)$ \\
\hline Dysuria and frequency & $25(46)$ & 149(47) & $62(52)$ \\
\hline Dysuria and urgency & $17(31)$ & 106(33) & $57(49)$ \\
\hline Dysuria and suprapubic pain & $11(20)$ & $57(18)$ & $32(27)$ \\
\hline Flank pain & $4(7)$ & $53(17)$ & $17(14)$ \\
\hline Genital symptoms & $4(7)$ & $30(9)$ & $16(13)$ \\
\hline Reported Fever & $1(2)$ & $27(8)$ & $9(7)$ \\
\hline Offensive smell & $6(11)$ & $57(18)$ & $26(22)$ \\
\hline Macrohematuria & $4(7)$ & $31(10)$ & $17(15)$ \\
\hline UTI within 4 weeks & 0 & $18(6)$ & $14(12)$ \\
\hline Other $^{a}$ & $6(11)$ & $27(8)$ & 15(13) \\
\hline Anamnestic score ${ }^{b}$ & $2(2 ; 3)$ & $3(2 ; 4)$ & $3(2 ; 4)$ \\
\hline
\end{tabular}

Figures are $\mathrm{n}(\%)$, if not marked otherwise ${ }^{\text {a }}$ Signs and symptoms not listed in the questionnaire

${ }^{\mathrm{b}}$ Median IQR (Interquartile range) of signs, symptoms and anamnesis of previous UTI (Score 1 to 11)

\section{Diagnostic workup and treatment decisions}

Table 3 shows the distribution of appropriate treatment decisions in the three diagnostic pathway groups. The distribution of the treatment decision was different between the three diagnostic pathways. For example, the day of the index consultation (i.e. first treatment decision), overtreatment was seen in $39 \%$ of patients without culture, $14 \%$ of those with culture in practice, and $27 \%$ of the patients with culture sent to hospital ( $p$-value: 0.0001$)$.
For the final treatment decision, an appropriate treatment decision was taken in: $55 \%$ of patients without culture, $71 \%$ of those with culture in practice, and $69 \%$ of the patients with cultures sent to hospital $(p$-value $=0.04)$.

\section{Discussion}

\section{Summary of results}

In a practice setting with wide availability of diagnostic tests, nearly all patients with suspected UTI are examined

Table 3 Appropriateness of the treatment decision and use of diagnostic tests

\begin{tabular}{|c|c|c|c|c|c|c|}
\hline & \multicolumn{3}{|c|}{ First treatment decision $(p \text {-value }=<0.0001)^{a}$} & \multicolumn{3}{|c|}{ Final treatment decision $(p \text {-value }=0.04)^{b}$} \\
\hline & Appropriate & Overtreatment & Undertreatment & Appropriate & Overtreatment & Undertreatment \\
\hline $\begin{array}{l}\text { No culture } \\
(n=54)\end{array}$ & $30(55)$ & 21(39) & $3(6)$ & $30(55)$ & $21(39)$ & $3(6)$ \\
\hline Culture performed in practice $(n=317)$ & $176(56)$ & $46(14)$ & $95(30)$ & $227(71)$ & $76(24)$ & $14(5)$ \\
\hline Culture performed at hospital $(n=117)$ & $71(61)$ & $32(27)$ & $14(12)$ & $81(69)$ & $24(21)$ & $12(10)$ \\
\hline
\end{tabular}

Appropriate $=$ prescribing antibiotics when reference test is positive and not prescribing antibiotics when reference test is negative Ovrertreatment $=$ prescribing antibiotics when reference test is negative Undertreatment $=$ not prescribing antibiotics when reference test is positive

Permutation LR test to assess whether treatment decision at the day of the index consultation ${ }^{\mathrm{a}}$ or at the day of the final treatment decision ${ }^{\mathrm{b}}$ differs between groups: a) no culture, b) culture performed in practice, c) culture performed at hospital. Hierarchical model with practices as a random intercept and adjusted for: complicated versus uncomplicated UTI, offensive smell, anamnesis score, microscope and culture in practice availability

'When not performing culture, first treatment decision is the final treatment decision 
with urine dipstick and for most, urine culture is part of the diagnostic workup. Neither a suspected complicated UTI nor the results of dipstick or microscopy predict the incorporation of urine culture within the diagnostic workup. Incorporation of urine culture within the decision-making process is associated with a higher proportion of appropriate treatment decisions.

\section{Strengths and limitations}

Although a randomized controlled trial might be considered the ideal design to assess the effect of diagnostic tests on treatment decisions, there is no consensus about the best study design to help understand the link between combination of diagnostic test, test interpretation and treatment decision $[6,13,14]$. Since our study aimed at describing the use of diagnostic tests and the appropriateness of the treatment decision under daily practice conditions, a prospective observational design was a sensible way of capturing the whole decision-making process from consultation to final treatment decision [15].

The observational study was carried out as part of the context assessment of a quality improvement program. It brings about some advantages and disadvantages.

On one hand, the external validity needs to be considered with caution. Only 39 practices out of 500 accepted to participate. Previous studies have shown that GPs that participate in research or quality improvement activities tend to prescribe fewer antibiotics and are possibly more interested in reducing inappropriate prescribing than their non-participating colleagues [16, 17]. Therefore, the differences shown in this study may be conservative.

On the other hand, observation bias was minimized by the fact that GPs were interested in obtaining an accurate recording of their decisions under daily practice conditions. This type of medical audit has been extensively used in Denmark for more than 25 years [18]. The cornerstone of the methodology is the bottom-up approach in which the GPs set their own improvement goals. Hence, there is no point to record data that do not reflect their everyday practice.

Finally, we cannot completely rule out residual confounding due to unmeasured variables. Nonetheless, the effect of clustering was considered in the assessment of predictors and differences between the diagnostic groups. The model to test the association between the diagnostic pathway groups and treatment decision was adjusted by the predictors for the combination of tests and differences in baseline characteristics between the diagnostic pathway groups.

\section{Interpretation of the findings in relation to previous literature}

This study shows the complexity of the decision-making process in a group perceived as "easy" patients in general practice [19].
International guidelines recommend the inclusion of urine culture as part of the diagnostic process only in suspected complicated UTI to target the right antibiotic choice [20, 21]. In our study, an uncomplicated versus complicated UTI did not predict the incorporation of urine culture within the diagnostic workup. It may be explained by the current debate on finding a new approach [22] to define the type of UTI based on burden of symptoms, risk factors, and availability of appropriate antimicrobial therapy. For example, in the no culture group the median anamnestic score was lower than in the other two groups. It means GPs possibly considered these patients as "uncomplicated cases". Furthermore, the higher the anamnestic score the higher the probability to send the urine culture to the hospital and the lower the probability to perform the urine culture in the practice.

Results from the tests performed previously did not predict incorporation of a test within the diagnostic work-up. It indicates a quality problem in the use of diagnostic tests. An obvious reason for the unsystematic use of diagnostic tests might be the payment scheme. Danish primary care system provides economic incentive for the GP to perform laboratory testing in practice, and this may have influenced the diagnostic workup.

Nonetheless, shortcomings of the dipstick and microscopy may lead to the incorporation of another test in order to gain certainty on the diagnosis. The use of dipsticks in primary care needs to take into consideration two important factors for the interpretation [11]. Firstly, a minimum bladder incubation time of $4 \mathrm{~h}$, what is difficult if we take into consideration that one of the main motives of consultation is frequency. Secondly, common uropathogens such as Enterococcus spp. and Staphylococcus spp. do not reduce nitrites. Microscopy requires expertise and the sensitivity is low with colony counts $<10^{5}$ [23]. Then, Danish GPs may rely more on the results of a urine culture.

Behavioural and medical reasons may explain the inclusion of urine culture in the diagnostic workup for most patients too.

Sackett et al. have long ago described four diagnostic approaches in clinical practice [24]. A mixture of the hypothetico-deductive strategy where the clinician gathers information to either confirm or refute his/her hypothesis and the complete history strategy could explain our results.

In a country with a strong antibiotic stewardship program, GPs are aware of the societal consequences of unnecessary use of antibiotics. They need to find a way to counteract the systematic error called "confirmation bias" [25]. This error arises from the tendency to seek and interpret evidence that predominantly confirms a pre-existing hypothesis. In our population, patients entered the study with a suspected UTI, thus for the GPs it may be difficult to withhold antibiotic prescribing. Not surprisingly, inappropriate use of antibiotics is mainly driven by overprescribing of antibiotics and only to a lesser extent by underprescribing. 
Cardinal symptoms such as dysuria, frequency and urgency has a high negative predictive value [26]. It means, they are useful to rule out a UTI when absent. However, in our study population, one of the inclusion criteria was dysuria and/or frequency so the negative predictive value could not be used to rule out a UTI.

Urine culture performed in a laboratory is currently regarded as the best available test to rule in or out an infection [23]. Thus, GPs may see the use of urine culture as the best way to counteract "the confirmation bias".

The clinical history plays a role when GPs decide whether to perform a urine culture in the practice or send it to the hospital. This has an effect as well on treatment decision.

In patients whose urine culture was performed in practice, the expectation of obtaining a result the following day combined with low anamnestic scores encouraged both GPs and patients to withhold antibiotics. The day of the index consultation, only $14 \%$ of patients were over-treated. However, incorrect interpretation of the culture resulted in $24 \%$ overtreatment for the final treatment decision.

For patients with hospital culture, a few days' delay of culture result combined with a higher symptom score led to more antibiotic overprescribing at the day of the index consultation (27\%). It is in line with a qualitative study on TDs in patients with suspected UTI, where GPs preferred to treat empirically due to the discomfort experienced by the patient [19].

\section{Perspectives}

Implementation of effective interventions to reduce inappropriate use of antibiotics requires thorough knowledge of the context in which the decision to prescribe antibiotics is taken [27]. For example, for the group of GPs that participated in this study, education on interpretation of urine culture in practice or improving communication with the microbiology department are interventions that might reduce the inappropriate prescribing of antibiotics.

This assessment should encourage the implementation of studies to assess the use of the available diagnostic tests to set-up improvement strategies that can reduce the unnecessary prescription of antibiotics within each context/country.

Finally, the results presented in this paper focus on the impact on treatment decision. Further research assessing the cost-effectiveness and impact on patients' outcomes should contribute with information to evaluate the multi-dimensional value of these diagnostic strategies.

\section{Conclusion}

In a context with wide availability of diagnostic tests, GPs use diagnostic tests in all the patients with suspected UTI. It may be explained by economic as well as medical/behavioural reasons. Inclusion of urine culture as part of the diagnostic workup leads to appropriate treatment decision in the majority of the patients. Performance of urine culture is important for reducing inappropriate prescribing in patients with suspected UTI seeking care in general practice in Denmark.

\section{Additional file}

Additional file 1: Table S1. Predictors for using diagnostic tests in patients with suspected UTI. Summary of the results of the logistic regression models to test the predictors for using microscopy, urine culture in practice or urine culture at the hospital. Table S2. Accuracy of urine culture in practice. Distribution of the interpretation of the test in relation to the reference standard. Table S3.

Accuracy of urine culture in the hospital. Distribution of the interpretation of the test in relation to the reference standard. (DOCX $22 \mathrm{~kb}$ )

\section{Abbreviations}

AMR: Antimicrobial resistance; GP: General Practitioner; NPV: Negative predictive value; PPV: Positive predictive value; SSI: Statens Serum Institute; TD: Treatment decision; UTI: Urinary tract infection; WHO: World Health Organization

\section{Acknowledgements}

We would like to acknowledge the General Practitioners and patients that contributed with data for the study. We would like to thank as well Anette Hammerum, Frank Hansen and Nadia Xenia Olsen for their work in the processing of the urine culture at the Statens Serum Institute (SSI). Finally, our deep gratitude to the Audit Project Odense (APO) group for data management.

\section{Funding}

This study was fund by: a) 2016 fund (grant from the University of Copenhagen to promote interdisciplinary research), b) KAP-H (agency for quality in primary care at the capital region), c) læge Sofus Carl Emil Friis og Hustru Olga Doris Friis' legat.

\section{Availability of data and materials}

The datasets used and/or analysed during the current study are available from the corresponding author on reasonable request.

\section{Authors' contributions}

GC AH TMS MM LB NF designed the study. VS HS supervised the statistical analysis and contributed with the interpretation. GC wrote the first draft and all authors critically revised the manuscript and approved the final version.

Ethics approval and consent to participate

The study was approved by The National Committee on Health Research Ethics of Denmark (case number: H-4- 2014-097). Informed written consent was obtained from all patients participating in the study.

\section{Competing interests}

The authors declare that they have no competing interests.

\section{Publisher's Note}

Springer Nature remains neutral with regard to jurisdictional claims in published maps and institutional affiliations.

\section{Author details}

${ }^{1}$ The Research Unit for General Practice and Section of General Practice, Department of Public Health, University of Copenhagen, Øster Farimagsgade 5, 1014 Copenhagen, Denmark. ${ }^{2}$ Department of Veterinary Clinical Sciences, University of Copenhagen, Copenhagen, Denmark. ${ }^{3}$ Finnish Office for Health Technology Assessment (FINOHTA) - National Institute for Health and Welfare, Helsinki, Finland. ${ }^{4}$ Department of Clinical Microbiology, Rigshospitalet, Copenhagen, Denmark. 
Received: 10 June 2017 Accepted: 1 May 2018

Published online: 16 May 2018

\section{References}

1. World Health Organization. Global action plan on antimicrobial resistance. 2015. http://www.who.int/antimicrobial-resistance/publications/globalaction-plan/en/.

2. Adriaenssens $S$, Versporten A, Muller A, Minalu G, Faes C, Vankerckhoven V, et al. European surveillance of antimicrobial consumption (ESAC): outpatient antibiotic use in Europe (1997-2009). J Antimicrob Chemother. 2011;66 Suppl 6:vi3-12.

3. Statens Serum Institut. DANMAP 2015-use of antimicrobial agents and occurrence of antimicrobial resistance in bacteria from food animals, food and humans in Denmark. 2015. http://www.danmap.org/pdf.ashx?title= Reports\&url=http\%3A\%2F\%2Fwww.danmap.org\%2FDownloads\%2FReports. aspx\%3Fpdf\%3D1.

4. Aabenhus R, Hansen MP, Siersma V, Bjerrum L. Clinical indications for antibiotic use in Danish general practice: results from a nationwide electronic prescription database. Scand J Prim Health Care. 2017;35:162-9.

5. Haldrup S, Thomsen RW, Bro F, Skov R, Bjerrum L, Søgaard M. Microbiological point of care testing before antibiotic prescribing in primary care: considerable variations between practices. BMC Fam Pract. 2017;18:9.

6. Bossuyt PM, Lijmer JG, Mol BW. Randomised comparisons of medical tests: sometimes invalid, not always efficient. Lancet. 2000;356:1844-7.

7. Thompson M, Weigl B, Fitzpatrick A, Ide N. More than just accuracy: a novel method to incorporate multiple test attributes in evaluating diagnostic tests including point of care tests. IEEE J Transl Eng Heal Med. 2016;4:2800208.

8. Hulsher ME, Meer JW, Grol RP. Antibiotic use: how to improve it? Int JMed Microbiol. 2010;300:351-6.

9. Teixeira Rodrigues A, Roque F, Falcão A, Figueiras A, Herdeiro MT. Understanding physician antibiotic prescribing behaviour: a systematic review of qualitative studies. Int J Antimicrob Agents. 2013;41(3):203-12.

10. Cordoba G, Sorensen T, Holm A, Bjornvad C, Bjerrum L, Jessen L. Exploring the feasibility and synergistic value of the one health approach in clinical research: protocol for a prospective observational study of diagnostic pathways in human and canine patients with suspected urinary tract infection. Pilot Feasibility Stud. 2015:1:38.

11. European confederation of laboratory medicine. European urinalysis guidelines. Scand J Clin Lab Invest Suppl. 2000;231:1-86.

12. Bates $D$, Mächler $M$, Bolker $B$, Walker $S$. Fitting linear mixed-effects models using Ime4. J Stat Soft. 2015;67:48.

13. Kennedy AG. Evaluating diagnostic tests. J Eval Clin Pract. 2016;22:575-9.

14. Trikalinos TA, Siebert U, Lau J. Decision-analytic modeling to evaluate benefits and harms of medical tests: uses and limitations. Med Decis Mak. 2009;29(5):E22-9.

15. Home P, Yang W, Zilov A, Soewondo P, Bech OM, Sekkal F, et al. How can observational trials inform and improve clinical practice? Observational studies: going beyond the boundaries of randomized controlled trials. Diabetes Res Clin Pract. 2010;88:S3-9.

16. Strandberg EL, Ovehed I, Troein M, Hakansson A. Influence of selfregistration on audit participants and their non-participating colleagues. A retrospective study of medical records concerning prescription patterns. ScandJPrimHealth Care. 2005;23(1):42-6.

17. Akkerman AE, Kuyvenhoven MM, Verheij TJ, Van Dijk L. Antibiotics in Dutch general practice: nationwide electronic GP database and national reimbursement rates. Pharmacoepidemiol Drug Saf. 2008:17(4):378-83.

18. Munck AP, Hansen DG, Lindman A, Ovhed I, Forre S, Torsteinsson JB. A Nordic collaboration on medical audit. The APO method for quality development and continuous medical education (CME) in primary health care. Scand J Prim Heal Care. 1998;16:2-6.

19. Duane S, Domegan C, Callan A, Galvin S, Cormican M, Bennett K, et al. Using qualitative insights to change practice: exploring the culture of antibiotic prescribing and consumption for urinary tract infections. BMJ Open. 2016;6:e008894

20. Scotish Intercollegiate Guidelines Network. Management of suspected bacterial urinary tract infection in adults: a national clinical guideline. 2015. http://www.sign.ac.uk/quidelines/fulltext/88/.

21. Gupta K, Hooton TM, Naber KG, Wullt B, Colgan R, Miller LG, et al. International clinical practice guidelines for the treatment of acute uncomplicated cystitis and pyelonephritis in women: a 2010 update by the
Infectious Diseases Society of America and the European Society for Microbiology and Infectious Diseases. Clin Infect Dis. 2011;52(5):e103-20.

22. Johansen TE, Botto H, Cek M, Grabe M, Tenke P, Wagenlehner FM, et al. Critical review of current definitions of urinary tract infections and proposal of an EAU/ESIU classification system. Int J Antimicrob Agents. 2011;38:64-70.

23. Schmiemann G, Kniehl E, Gebhardt K, Matejczyk MM, Hummers-Pradier E. The diagnosis of urinary tract infection: a systematic review. Dtsch Arztebl Int. 2010;107:361-7.

24. Sacket D, Haynes RB, Tugwell P, Guyatt G. In: 2nd, editor. Clinical epidemiology: a basic sciences for clinical medicine. New York: Little, Brown and Company; 1991

25. Bornstein BH, Emler AC. Rationality in medical decision making: a review of the literature on doctors' decision-making biases. J Eval Clin Pract. 2001;7: 97-107.

26. Giesen LG, Cousins G, Dimitrov BD, van de Laar FA, Fahey T. Predicting acute uncomplicated urinary tract infection in women: a systematic review of the diagnostic accuracy of symptoms and signs. BMC Fam Pr. 2010;11:78,

27. Arnold SR. Straus SE. Cochrane Database Syst Rev: Interventions to improve antibiotic prescribing practices in ambulatory care; 2005. http://www.ncbi. nlm.nih.gov/pubmed/16235325

\section{Ready to submit your research? Choose BMC and benefit from:}

- fast, convenient online submission

- thorough peer review by experienced researchers in your field

- rapid publication on acceptance

- support for research data, including large and complex data types

- gold Open Access which fosters wider collaboration and increased citations

- maximum visibility for your research: over $100 \mathrm{M}$ website views per year

At BMC, research is always in progress.

Learn more biomedcentral.com/submissions 